\title{
communications biology
}

ARTICLE

https://doi.org/10.1038/s42003-022-03059-8

OPEN

\section{The C-terminal tail of $\alpha$-synuclein protects against aggregate replication but is critical for oligomerization}

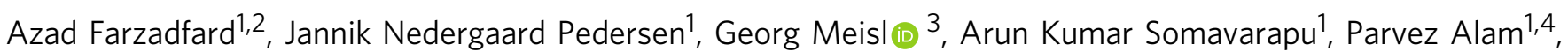
Louise Goksøyr (10 5, Morten Agertoug Nielsen (1) ${ }^{5}$, Adam Frederik Sander (1) 5, Tuomas P. J. Knowles (D) ${ }^{3,6}$, Jan Skov Pedersen (1) ${ }^{1,7}$ \& Daniel Erik Otzen (1) ${ }^{1,8}{ }^{凶}$

Aggregation of the 140-residue protein $\alpha$-synuclein $(\alpha \mathrm{SN})$ is a key factor in the etiology of Parkinson's disease. Although the intensely anionic C-terminal domain (CTD) of $\alpha \mathrm{SN}$ does not form part of the amyloid core region or affect membrane binding ability, truncation or reduction of charges in the CTD promotes fibrillation through as yet unknown mechanisms. Here, we study stepwise truncated CTDs and identify a threshold region around residue 121; constructs shorter than this dramatically increase their fibrillation tendency. Remarkably, these effects persist even when as little as $10 \%$ of the truncated variant is mixed with the fulllength protein. Increased fibrillation can be explained by a substantial increase in self-replication, most likely via fragmentation. Paradoxically, truncation also suppresses toxic oligomer formation, and oligomers that can be formed by chemical modification show reduced membrane affinity and cytotoxicity. These remarkable changes correlate to the loss of negative electrostatic potential in the CTD and highlight a double-edged electrostatic safety guard.

\footnotetext{
${ }^{1}$ Interdisciplinary Nanoscience Center (iNANO), Aarhus University, Gustav Wieds Vej 14, 8000 Aarhus C, Denmark. ${ }^{2}$ School of Biology, College of Science, University of Tehran, Tehran, Iran. ${ }^{3}$ Yusuf Hamied Department of Chemistry, University of Cambridge, Lensfield Road, Cambridge CB2 1EW, UK. ${ }^{4}$ Department of Biomedicine, Aarhus University, 8000 Aarhus C, Denmark. ${ }^{5}$ Centre for Medical Parasitology at the Department of Immunology and Microbiology, University of Copenhagen, Blegdamsvej 3B, 2200 Copenhagen N, Denmark. ${ }^{6}$ Cavendish Laboratory, University of Cambridge, J J Thomson Avenue, Cambridge CB3 OHE, UK. ${ }^{7}$ Department of Chemistry, Aarhus University, Langelandsgade 140, 8000 Aarhus C, Denmark. ${ }^{8}$ Department of

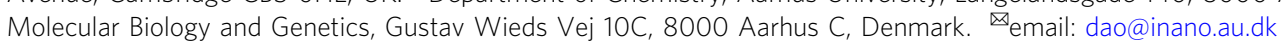


A lpha-synuclein $(\alpha \mathrm{SN})$ is a 140-residue intrinsically disordered protein, mainly expressed in brain cells ${ }^{1}$. Aggregation of aSN to form insoluble fibrils and smaller oligomeric species ( $\alpha \mathrm{SOs}$ ), a process which is increased in different familial variants of Parkinson's disease (PD) ${ }^{2}$, is critical to the onset and development of PD. This involves neuronal toxicity propagated through the brain, presumably via the transmission of aSN aggregates from cell to cell ${ }^{1}$. Thus, control of aSN aggregation and transmission may eventually lead to therapies against PD.

aSN can be divided into 3 regions. The $\mathrm{N}$-terminal domain (NTD, residues 1-60) mediates binding to synaptic vesicles, which is believed to contribute to aSO toxicity ${ }^{3,4}$. The NAC domain (residues 61-95) forms the core folded region of both $\alpha \mathrm{SOs}^{5}$ and fibrils ${ }^{6,7}$. Finally, the highly negatively charged C-terminal domain (CTD, residues 96-140), which remains mobile both in $\mathrm{aSOs}^{5,8}$ and fibrils $s^{6,7}$, can also bind to synaptic vesicles through bridging $\mathrm{Ca}^{2+}$ ions ${ }^{9,10}$. The CTD's high mobility and accessibility in all $\alpha \mathrm{SN}$ species makes CTD truncation of $\alpha \mathrm{SN}$ a relatively common occurrence in vivo. Truncations at residues $103,110,113,114,115,119,122,124,125,133$, and 135 have been identified in vivo ${ }^{11}$. Those at 119 and 122 at most prevalent and occur at $20-25 \%$ abundance relative to full-length aSN. The truncations typically result from incomplete degradation of fulllength $\alpha \mathrm{SN}$ by the proteasome ${ }^{12}$ or other proteases like Calpain $\mathrm{I}^{13}$. CTD truncation is linked to PD, though the exact causality is unclear. CTD truncation produced by alternative splicing in response to oxidative stress results in proteosomal dysfunction and cell death ${ }^{14}$. Transgenic mice and cells expressing aSN mutants linked to familial PD produce CTD-truncated $a \mathrm{SN}^{15}$. Co-expression of CTD-truncated $\alpha \mathrm{SN}$ in rats induces accumulation and aggregation of full-length aSN and impaired dopamine release $^{16}$. CTD-specific antibodies ameliorate neurodegeneration pathology and behavior in PD-mice model and inhibit disease propagation ${ }^{17}$.

Besides these compelling cellular data, CTD truncation accelerates aSN fibrillation in vitro by decreasing the fibrillation lag time and increasing the extent of fibrillation ${ }^{18-21}$. The effect is proportional to the removal of negative charge, indicating that full-length CTD may suppress aggregation through long-range interactions with the $\mathrm{NAC}^{22}$ or N-terminal region ${ }^{23}$. Fibrillation is accelerated by screening the CTD's negative charges through cations ${ }^{20}$ or lowered $\mathrm{pH}^{19}$. CTD truncation also leads to twisted, thinner, and shorter fragmented fibrils ${ }^{21,24,25}$. This may reflect decreased charge repulsion between individual fibrils and increased exposure of hydrophobic surface area and intermolecular $\beta$-sheets ${ }^{23}$.

$\alpha$ SOs likely exert toxicity by vesicle binding, leading to synaptic impairment and neuronal dysfunction ${ }^{26-28}$. Studies are challenged by many types of reported $\mathrm{aSO}^{29}$. However, the most prevalent species (formed in PBS buffer at $37^{\circ} \mathrm{C}$ and high concentrations of $\alpha \mathrm{SN}$ ) assumes an ellipsoidal core-shell structure, in which a compact $\beta$-sheet rich core (the NAC region and part of the NTD) is surrounded by a shell of dynamic residues (mostly the CTD $\left.{ }^{5}\right)^{29-32}$. This species is highly stable ${ }^{33}$ and weakly blocks aSN fibrillation ${ }^{32}$, indicating that it is an off-pathway species. Similar aSOs form with PD-related lipid oxidation products such as 4-oxo-2-nonenal $(\mathrm{ONE})^{34}$. Despite its dynamic nature, the CTD also plays an important role in the formation of aSOs. For instance, its interactions with $\mathrm{Cu}^{2+}$ and $\mathrm{Ca}^{2+}$ induces $\alpha \mathrm{SO}$ formation 35,36 . The neurotransmitter dopamine inhibits aSN aggregation and stabilizes aSOs by interacting with the motif $\mathrm{Y}_{125}$ EMPS $_{129}$ in the CTD ${ }^{37,38}$.

It remains unclear how CTD truncations affect the molecular mechanism of fibrillation and the formation and properties of oligomeric species. Using a series of truncation mutants we show that removal of more than 15 residues strongly enhances fibrillation thanks to increased rate of replication via a secondary process (fragmentation or secondary nucleation). Remarkably, oligomerization is essentially abolished unless ONE-assisted. These truncated oligomers are however larger, less stable, and much less disruptive towards vesicles and cells. Thus, the C-terminal tail plays a central role in aSN structure and function.

\section{Results}

C-terminal truncation accelerates secondary processes in aSN fibrillation. To correlate changes in self-assembly with C-terminal tail length, 9 different aSN constructs were designed in which the CTD was truncated in steps of 4-6 residues from full length $\alpha \mathrm{SN}$ to residue 100 . aSN-FL is full-length $\alpha \mathrm{SN}$ while e.g., aSN115 refers to the construct consisting of the first 115 residues of aSN (and thus lacking residues 116-140). Recombinant expression levels in $E$. coli diminished steeply for constructs aSN110 and shorter, declining to $20 \%$ (compared to full-length) for aSN100 (Fig. S1).

To probe how truncations alter aSN aggregation, we recorded the kinetics of aggregation of each construct at a range of monomer concentration ([monomer]) (Figs. S2 and S3) and compared with integrated rate laws using the web-based program Amylofit ${ }^{39}$ which allows to identify kinetic models based on microscopic mechanisms that best describe macroscopic aggregation behavior. SDS-PAGE analyses confirmed that the great majority of aSN had aggregated by the end of the reaction (Fig. S4). (Note that slightly incomplete levels of fibrillation will not affect the outcome of the analysis in Amylofit.) TEM demonstrated very similar architecture for the fibrils formed by the different truncation variants (Fig. S5). In a plot of the halftime of fibrillation versus [monomer] on a double logarithmic plot (Fig. 1), the slope provides the scaling exponent $\gamma$, which serves as a guide to the dominant mechanism of aggregate formation. A single linear trend implies that the same process dominates over the whole concentration range, while a change in slope implies a change in the rate determining mechanism ${ }^{40}$. Strikingly, the constructs fall into two groups. Group one ( $a \mathrm{SN} 125$ up to $a \mathrm{SN}-\mathrm{FL}$ ) shows a single linear correlation over the whole concentration range. In group two (shorter than aSN125), a second linear regime emerges at high concentrations with a flat slope, i.e., fibrillation half times become independent of [monomer]. This starts to become visible for aSN121 and is very prominent for aSN105 (aSN100 aggregated very rapidly and irreproducibly). This implies that the fibrillation mechanism changes at high concentrations for the shorter constructs, most likely due to saturation effects of elongation or primary nucleation, which decrease the [monomer] dependence of the rate determining steps ${ }^{40}$.

The fibrillation curves for the group one constructs are best described by a model in which fibrils elongate by monomer addition in a process which is first order in [monomer], and multiply by a secondary process independent of [monomer] and reaction order zero, such as fragmentation or saturated secondary nucleation, the latter consistent with previous observations ${ }^{41}$. Fits with different molecular aggregation mechanisms are shown for aSN-FL in Fig. S2 along with plots fitted to models with secondary processes for aSN135- $\alpha \mathrm{SN} 125$. For group two constructs, comparison of this model has to be extended to allow for saturation of the elongation process (Fig. S3). Considering the elongation process as a two-step reaction (i.e., binding of monomers to the end of fibrils and their conformational conversion to fibrils), saturation of elongation implies that at higher [monomer], the reaction no longer exhibits first order kinetics with respect to [monomer] and the rate-limiting step 

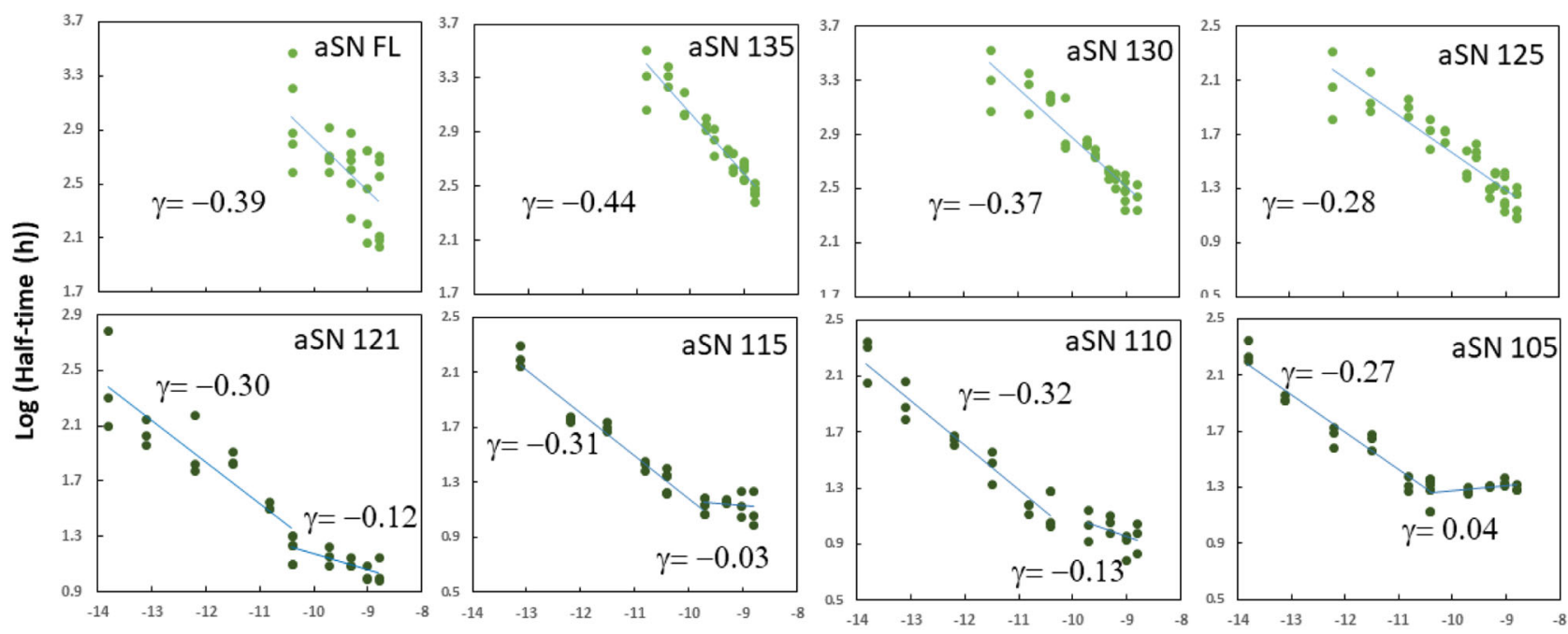

Log (monomer concentration (M))

Fig. 1 Effect of C-terminal truncation on $\boldsymbol{\alpha} \mathbf{S N}$ fibrillation. Log-log plots of fibrillation half-times versus concentrations whose slope provides the scaling exponent $\gamma$. The plots reveal a single linear correlation for $\alpha \mathrm{SN} 125$ and longer constructs and the growing prominence of a flat region at high protein concentration for shorter constructs. Each point represents the half-time of a ThT curve (i.e., the time to reach half the maximal fluorescence value) from a fibrillation experiment carried out at the indicated $\alpha \mathrm{SN}$ concentration.

shifts from monomer binding to the end of fibrils (which is dependent on [monomer]) to the conformational conversion of the monomer to the fibrillated state (which is not). These limiting cases correspond to the two linear regimes in Fig. 1.

As shown in Figs. S2 and S3, C-terminal truncation of aSN accelerates the fibrillation both by decreasing the lag time and by increasing the slope of the sigmoidal aggregation curves. We used global kinetic analysis to elucidate the mechanistic basis for this behavior. To compare rates of individual aggregation processes for different constructs, data for each construct were fitted with a model that includes a saturating elongation and a monomerindependent secondary process model. The rate constants describing primary nucleation $\left(k_{\mathrm{n}}\right)$, elongation $\left(k_{+}\right)$and secondary processes (fragmentation $k_{-}$or secondary nucleation $k_{2}$ ) are obtained as combinations (e.g., $k_{\mathrm{n}}{ }^{*} k_{+}$and $k_{-}{ }^{*} k_{+}$) from global fits of aggregation data in the absence of preformed seeds. To decompose the observed changes in these combined rate constants into changes in individual molecular rate constants, we determined elongation rates for the constructs using a fibrillation assay in the presence of high and variable amounts (5-25\%) of seeds with $50 \mu \mathrm{M}$ monomeric $\alpha \mathrm{SN}$. Under such conditions, the integrated rate laws exhibit a direct dependence on $\mathrm{k}_{+}$. The relative elongation rate constants for all constructs (from plots of the initial rate of fibrillation against the concentration of seed (Fig. S6) are all very similar and do not show any systematic variation with truncation (Fig. 2a) at $50 \mu \mathrm{M}$ monomer. We used these relative elongation rates together with the composite values to obtain relative rate constants of primary nucleation and of the secondary process (in both cases normalized against the value for aSN-FL). To compare different constructs with different reaction orders of nucleation, we calculated the nucleation rate at $50 \mu \mathrm{M}$, which is in the middle of the concentration range where the relative elongation rate was measured experimentally. There is a weak trend for primary nucleation rates to fall with construct length (Fig. 2b); more strikingly, we see a jump between two relatively constant levels in the rate of the secondary process (Fig. 2c) between aSN130 and aSN125 (we attribute the low $k_{\mathrm{n}}$ value of $\alpha \mathrm{SN} 115$ to a fitting artifact in which a low $k_{\mathrm{n}}$ value is compensated by a higher $k$. value). The increase in second rate constant reflects the sudden increase in the slopes of the sigmoidal curves between aSN130 and aSN125 (which the more noisy variation in primary nucleation rates does not) and demonstrates that the secondary process drives accelerated fibrillation in aSN125 to aSN105 (Fig. 2c).

This secondary process could either be fragmentation of growing fibrils or surface-catalyzed secondary nucleation (Fig. 2d). Distinguishing between saturated secondary nucleation and fragmentation can often be difficult. The scaling exponent $\gamma$ obtained from the $\log$ plot of fibrillation half-time versus [monomer] ${ }^{39}$ (Fig. 1) is comparable to -0.5 , the value obtained from theory for a system with fragmentation or saturated secondary nucleation, for all mutants ${ }^{39}$. We therefore compared aSN fibrillation in the presence of low concentrations of seeds, one under quiescent conditions and the other with shaking. Shaking has been shown to promote primary nucleation, by increasing the air-water interface, as well as fibril fragmentation ${ }^{42-44}$. Secondary nucleation is expected to be less dependent on shaking, though one could also envision an increase in the turnover rate, i.e., the rate of detachment of formed nuclei from the surface of fibrils and attachment of fresh monomer induced by agitation leading to an increase in the secondary nucleation rate. Our results are very clear: in the quiescent mode experiment, no ThT binding signal was observed after $96 \mathrm{~h}$, whereas fibrillation was completed after few hours in shaking mode for the truncated mutants and FL (Fig. 2e, f shows data for aSN121 while Fig. S7 shows the same behavior for other mutants as well as aSN FL). The fact that very low concentrations of seeds $(0.03 \%)$ strongly promoted the fibrillation demonstrates the dominance of secondary processes which, as outlined above, we believe is most likely agitation- induced fragmentation.

The accelerating effect persists with low levels of truncated aSN. To investigate the effect of co-incubating full-length and truncated $\alpha \mathrm{SN}$, we fibrillated a mixture of $\alpha \mathrm{SN}-\mathrm{FL}$ and $\alpha \mathrm{SN} 110$ at constant total concentration but variable proportions of the two proteins (Fig. $2 \mathrm{~g}$ ). Remarkably, as little as 10\% aSN110 shifted the fibrillation time course away from the slower fibrillating aSN-FL and closer to that of the faster fibrillating aSN110. The effect 


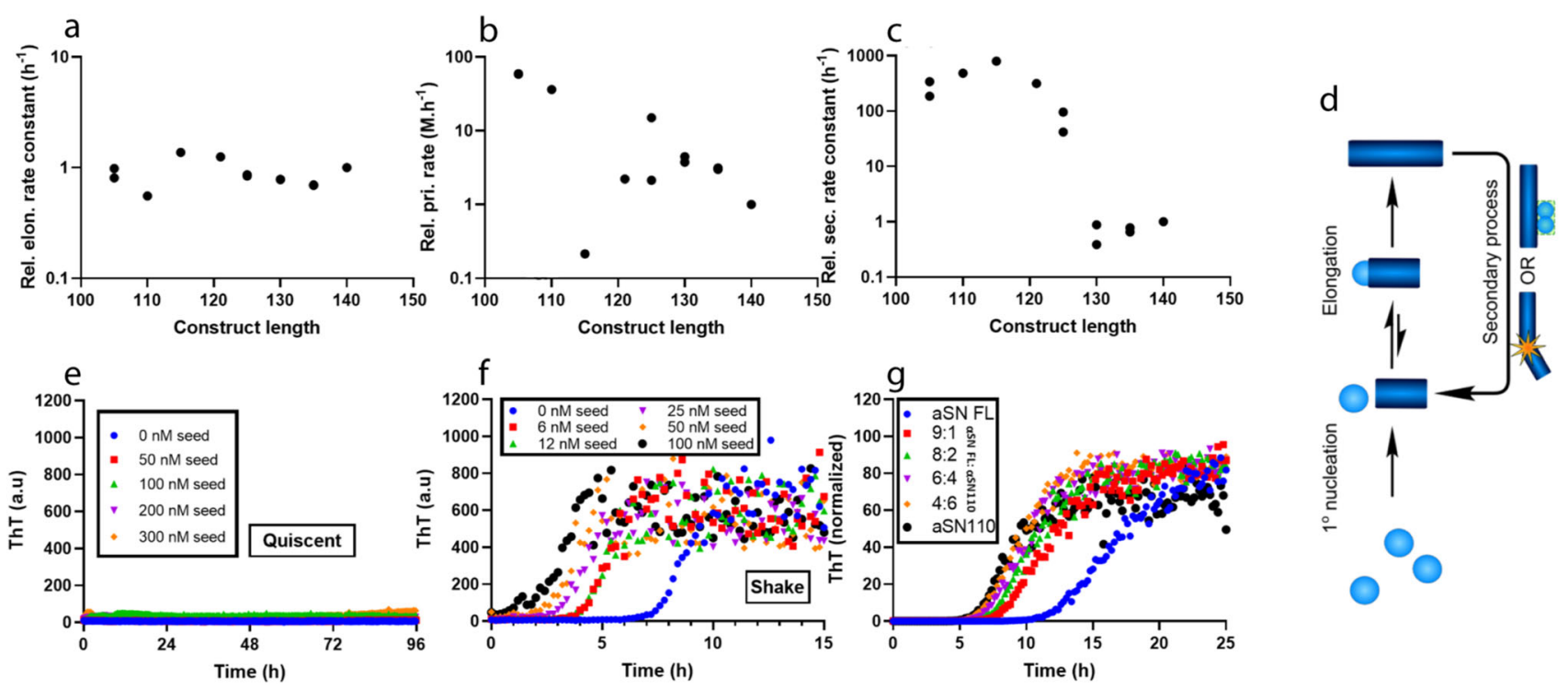

Fig. 2 Determination of individual rate constants of fibrillation for $\boldsymbol{\alpha} \mathbf{S N}$ truncated mutants. a Relative elongation rate constant, $k_{+}$, rel, obtained from seeded experiments shown in Fig. S6. There is little variation with length under the assumption that the length of the seed fibrils is similar for different truncation lengths. Details in Eq. 3. b The rate of primary nucleation, evaluated from the rate constant, $k_{n}$, and reaction order, $n_{c}$. Values calculated at $50 \mu \mathrm{M}$ $\alpha \mathrm{SN}$ to enable comparison between variants with varying reaction orders. c Rate constant of fragmentation, $k_{-}$, versus construct length. There is a very abrupt decrease between $\alpha \mathrm{SN} 125$ and $\alpha \mathrm{SN} 130$. Each data point in a-c corresponds to a separate experiment with three repeats in three wells; $\alpha \mathrm{SN} 105$, $\alpha S N 125$, aSN 130, and $\alpha S N 135$ were randomly repeated. d Schematic reaction network with an explicit two-step mechanism for elongation. e, $\mathbf{f}$ Low-seed $(0.03,0.06,0.1,0.2 \%)$ fibrillation without $(\mathbf{e})$ and with (f) shaking shows that shaking strongly promotes fibrillation. Data are for $\alpha \mathrm{SN} 121$. (g) ThT fibrillation of different mixtures of $\alpha \mathrm{SN}-\mathrm{FL}$ and $\alpha \mathrm{SN} 110$ (total protein concentration of $100 \mu \mathrm{M}$ ). Note that as little as $10 \% \alpha \mathrm{SN} 110$ significantly reduces the lag time. Shaking conditions in $\mathbf{f}, \mathbf{g}$ are reduced to $1 \mathrm{~min}$ (instead of the $10 \mathrm{~min}$ used for the other shaking experiments) in 12-min measurement cycles.

saturated quickly, and $60 \%$ aSN110 showed the same behavior as $100 \%$ aSN110. If truncated aSN mutants are more prone to fragmentation, then incorporation of even small amounts of these variants into a mixed fibril may disproportionately increase the rate of fragmentation. If instead truncation increases secondary nucleation, then the increased rate in the mixture may be due to the increased availability of defects and other catalytic sites on the fibril surface, believed to promote secondary nucleation. These results demonstrate the key importance of accounting for fast aggregating truncated versions, even when the full-length protein is the predominant form and truncated versions are present only in small proportions, as may be the case in vivo ${ }^{15,16}$.

Shorter constructs make bigger and longer aSOs which are less disruptive towards vesicles. To test the general tendency of CTD truncation mutants to form aSOs, we first optimized oligomerization conditions for aSN-FL by exploring parameters such as $\mathrm{NaCl}$ concentration, $\mathrm{pH}$, shaking, multiple rounds of freezedrying as well as the aSO inducing agent 4-oxononenal (ONE). Among these, multiple rounds of freeze-drying 45 and ONE modification $^{34}$ were most effective. aSOs accumulated in a linear fashion with each cycle of freeze-drying (Fig. S8a, b) without any change in structure according to Small Angle X-ray Scattering (Fig. S8c). Changes in $[\mathrm{NaCl}]$ did not help, but increasing $\mathrm{pH}$ to

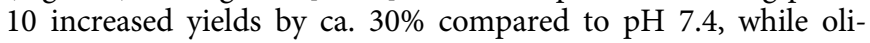
gomerization completely stopped around and below $\mathrm{pH} 6$ (Fig. S8d-f). The only residue expected to titrate around $\mathrm{pH} 6-7$ in $\mathrm{aSN}$ is the single His residue in position $50 \quad\left(\mathrm{pK}_{\mathrm{a}} \sim 6\right)$. Remarkably, the mutant His50Ala failed to oligomerize over a broad $\mathrm{pH}$ range, even after 3 cycles of freeze-drying (Fig. S8g).

Strikingly, while aSN121 and longer all gave the same yield of aSOs, based on the oligomer/monomer peaks height ratio (Fig. S8h, i), shorter constructs did not form any aSOs at all but remained monomeric even after three rounds of freeze-drying (Fig. S8h). These shorter constructs only formed aSOs with ONE
(Fig. S8j), leading to comparable yields of the main component oligomer (eluting around $12 \mathrm{ml}$ ) in all cases.

We investigated changes in shape and size of truncated aSOs by SAXS according to the previously developed model for aSN WT oligomer ${ }^{32}$. The core-shell model fitted both unmodified and ONE-modified truncated aSOs well (Fig. S9a, b). Pair distance distribution function $(p(r))$ curves showed an increase in maximal $r$-values with truncation, particularly for aSOs of length 115 and shorter (Fig. S9c, d). Correspondingly, truncation slightly increases radius of gyration in unmodified aSOs $\left(\mathrm{R}_{\mathrm{g}}\right)$ (Fig. 3a). In ONE-modified aSOs, changes in the long axis, shell thickness and $\mathrm{R}_{\mathrm{g}}$ (Fig. $3 \mathrm{~b}$ ) cluster in two groups: group one similar to ONEaSO-FL (ONE-aSO121 and longer) and group two with much bigger aSOs (shorter than aSO121). Again, truncation around 120 residues is critical. Group two consists of constructs unable to form aSO without ONE. The number of amino acid residues in the shell of ONE-modified aSOs decreased with truncation, while there was no clear trend for unmodified aSOs (Fig. 3c) (a trend towards more residues per shell was obscured by aSO121). For all aSOs, truncation increased the number of monomers per aSO (Fig. 3d).

There was only a very modest overall reduction in $\beta$-sheet content in ONE-modified aSOs (ONE- $\alpha \mathrm{SO}$ ) compared to unmodified aSOs (from 39 to $32 \%$ ), and the truncations do not significantly change aSO secondary structure (Fig. S9e-h). TEM images showed elongated ONE-modified aSOs but ring-shaped unmodified aSOs (Fig. S10). However, the shapes of truncated aSOs were similar to that of aSN-FL, and both aSOs and ONEmodified aSOs had diameters of $\sim 10 \mathrm{~nm}$. Oligomer size measured by TEM is similarly $\sim 10 \mathrm{~nm}$ for all the mutants, consistent with the core region being stained, while the more dynamic shell region remains unstained.

There is substantial evidence that permeabilization of cell membranes by aSOs leads to calcium ion influx and cell death $^{46-48}$. As a simple model system to mimic this phenomenon, 

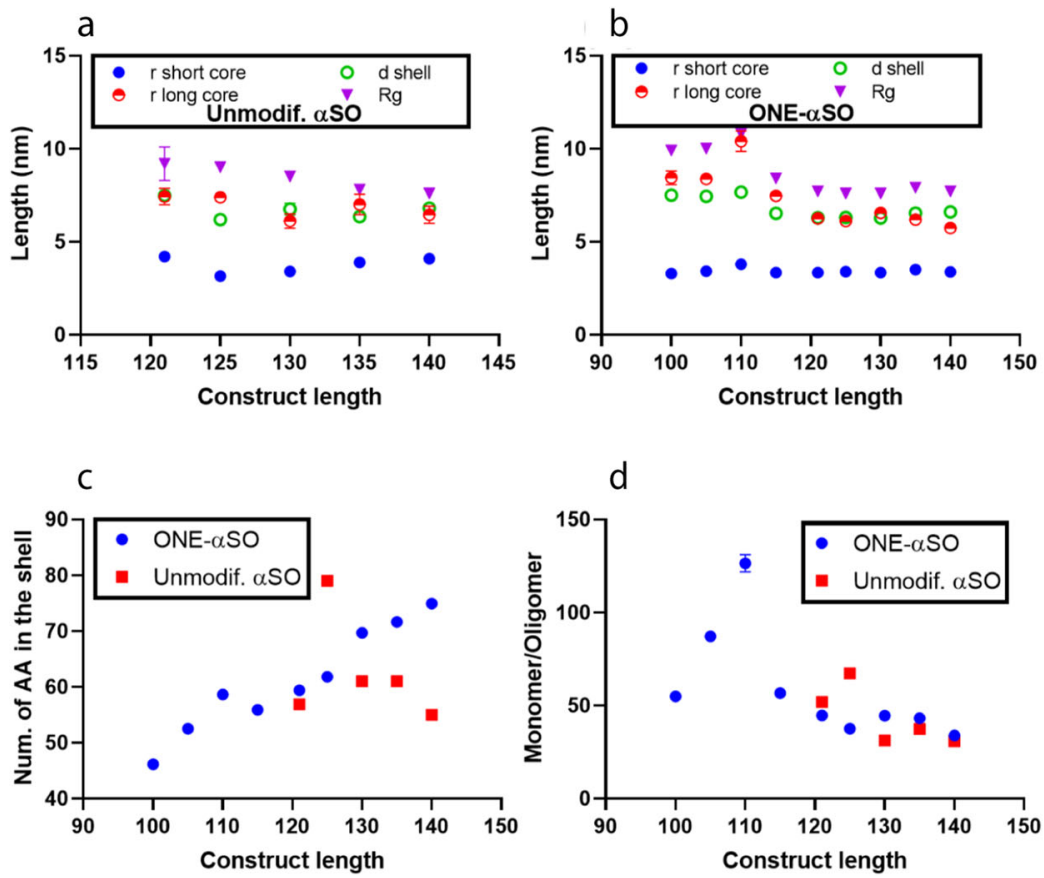

Fig. 3 Properties of ONE-modified and unmodified oligomers of truncated $\boldsymbol{\alpha} \mathbf{S N}$ mutants. a-d SAXS analysis of C-terminal truncated $\alpha \mathrm{SN}$ oligomers. $\mathbf{a}$, $\mathbf{b}$ The data with a previously developed model for $\alpha \mathrm{SO} 140$ suggest similar ellipsoidal shapes for all oligomers, i.e., a dense core and diffuse shell. However, parameters related to the shape of core and shell change in different ways in truncated constructs of unmodified versus ONE-modified $\alpha \mathrm{SO}$ s. c The number of amino acids in the shell fluctuate in unmodified $\alpha \mathrm{SO}$ s but decrease with truncation in ONE-modified $\alpha \mathrm{SO}$. $\mathbf{d}$ There is also an increase in the number of monomers per oligomer for both kinds of oligomers. Each point is the average of three replicates where error bars show standard deviations. e Schematic model of unmodified $\alpha S N$ FL oligomers based on SAXS ${ }^{32}$. A compact core is surrounded by a shell of more flexible polypeptide chains.

we determined the ability of these $\alpha \mathrm{SO}$ constructs to permeabilize negatively charged vesicles, quantified as the concentration of protein needed for $50 \%$ calcein release $\left(\mathrm{CR}^{50 \%}\right)$. Though all truncated aSN monomers were disruptive, $\mathrm{CR}^{50 \%}$ values increased significantly with truncation (Fig. 4a, b). Like their monomeric counterparts, truncation decreases aSO disruptiveness (Fig. 4c, d) but aSOs were more vesicle-disruptive than monomers. Strikingly, although calcein release from the longer ONE-modified aSOs (ONE- $\alpha$ SO125 and longer) was similar to unmodified aSOs, ONE-modified aSOs of shorter constructs (ONE- $\alpha \mathrm{SO} 100$ to ONE- $\alpha \mathrm{SO} 121$ ) showed reduced disruption (Fig. 4e, f). Gratifyingly, these results agreed with aSO cell toxicity assays on SH-SY5Y neuroblastoma cells, exposed for $24 \mathrm{~h}$ to aSOs (Fig. 4g). With the exclusion of aSO121, there is a good correlation between $\mathrm{CR}^{50 \%}$ values and cell viability (Fig. S11), supporting calcein release as a proxy for cell toxicity. Toxicity of unmodified aSOs was unaffected by truncation, whereas ONEmodified aSOs divide with statistical significance into longer aSOs with higher toxicity and shorter aSOs with lower toxicity (Fig. 4h).

\section{Discussion}

C-terminal truncation of aSN led to two conspicuous changes in fibrillation kinetics. Firstly, a saturation of the elongation step for aSN121 and shorter constructs. Secondly, a steep increase in the rate of the secondary process for aSN125 and shorter constructs led to faster fibrillation of truncated $\alpha \mathrm{SN}$. An increased rate of the secondary process, either through an increased tendency to fragment or (less likely) through an increased secondary nucleation rate, should lead to shorter average lengths of fibrils. Consistent with increased fragmentation, shorter C-terminal truncated constructs of $\alpha \mathrm{SN}$ are known to produce shorter fibrils ${ }^{21}$.
Critical role of the C-terminal 20 residues of aSN in modulating intramolecular contacts through electrostatic interactions. The effect of CTD on fibrillation is well-documented. Truncation of CTD at positions 103,118, and 129 or replacing anionic residues in CTD with neutral counterparts speeds up fibrillation ${ }^{49}$. CTD's protective role against fibrillation has been attributed to the interaction of the negatively charged CTD with the positively charged N-terminal and NAC regions ${ }^{22,23,50 .}$ Hydrogen exchange (HX) of the aSN monomer shows protection in the CTD that indicates transient long range interaction between CTD and NTD ${ }^{51}$. These interactions can be screened by $\mathrm{Ca}^{2+}$ ions that bind to CTD and increase solvent accessibility of NTD, resulting in a higher aggregation propensity ${ }^{50}$. A systematic analysis of the electrostatic repercussions of truncation is illuminating. The electrostatic potential of aSN remains essentially zero for the first 100 residues, after which it rises to a plateau from residue 120 onward $^{52}$. Together with our data, these findings indicate a critical role for the C-terminal 20 residues, which likely interact with the NTD to regulate aSN fibrillation and oligomer formation. Removal of the residues with higher electrostatic potential frees up the NTD/NAC region for intermolecular contacts. In support of this, NAC-derived peptides bind to the CTD and accelerate aggregation, probably by blocking NAC-CTD contacts ${ }^{53}$. As further evidence that CTD contacts with NTD/NAC can block fibrillation, mutants containing intramolecular disulfide bonds between residues 107 and 124 in CTD combined with intramolecular disulfide bonds in the NTDNAC region (residues 9, 42, 69, and 89) do not fibrillate; by contrast disulfide bonds in the NTD-NAC region alone are insufficient to inhibit fibrillation ${ }^{54}$.

CTD truncation introduces a saturation step in elongation. Faster elongation in CTD truncated constructs (caused by loss of 

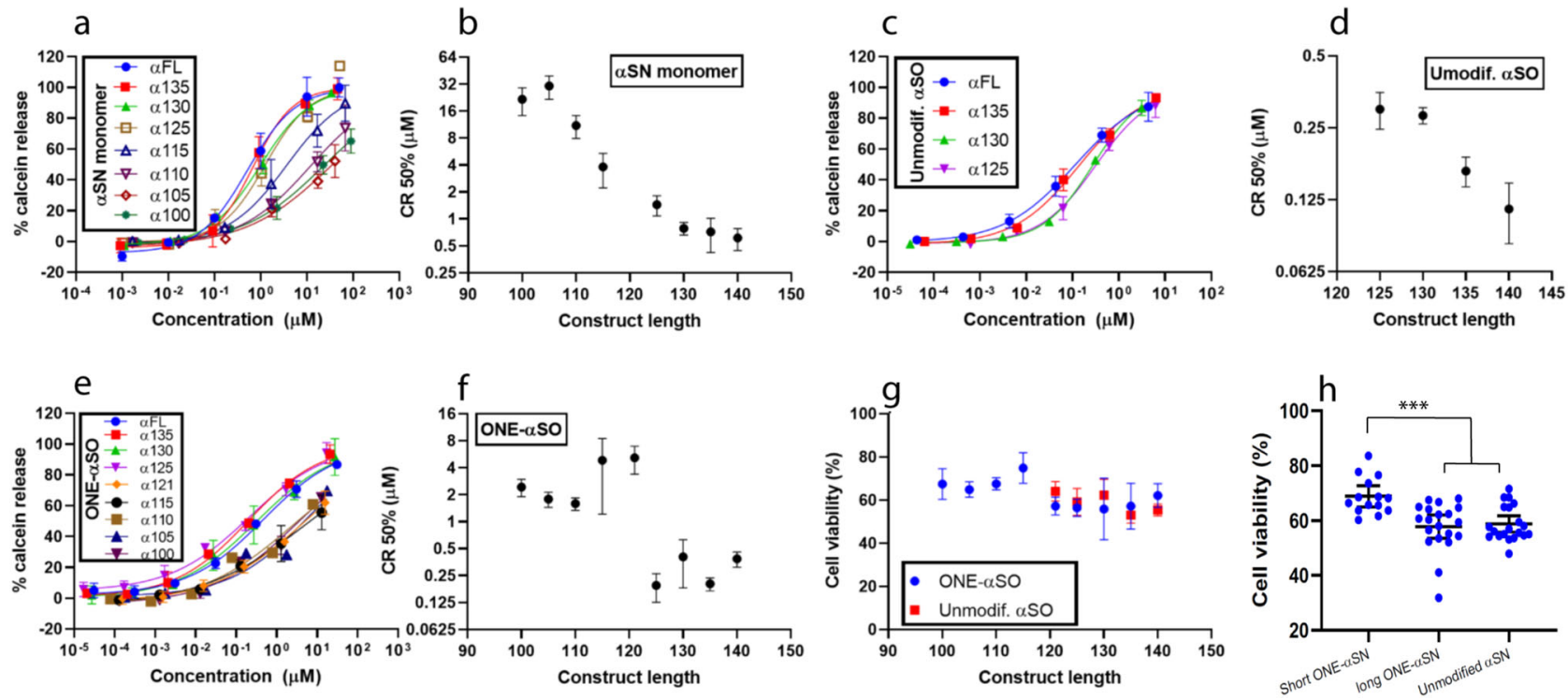

Fig. 4 Vesicle permeabilization and cell toxicity properties of CTD truncated $\boldsymbol{\alpha} \mathbf{S N}$ and their oligomers. a-f $\vee$ esicle permeabilization by truncated $\alpha \mathrm{SN}$ constructs. Permeabilization assays with truncated $\alpha \mathrm{SN}$ monomers (a, $\mathbf{b})$, unmodified $\alpha \mathrm{SO}$ s (c, $\mathbf{d})$, and ONE-modified $\alpha \mathrm{SN}$ monomers (e, $\mathbf{f}$ ) shows more aggressive behavior for oligomers in comparison to monomers, however, ONE- $\alpha \mathrm{SO} 121$ and the shorter ones are not as aggressive as the ONE- $\alpha \mathrm{SO} 125$ and longer oligomers. $\mathbf{g}, \mathbf{h}$ Cell viability with $10 \mu \mathrm{M}$ oligomer confirms the results of vesicle permeabilization. In $\mathbf{h} \alpha \mathrm{SO}$ s of $\alpha \mathrm{SN} 121$ and longer variants are binned into one group and $\alpha \mathrm{SN} 115$ and shorter variants into another. Cell toxicity of each group was compared by ANOVA (in graph pad software). ${ }^{\star \star \star} p$ value $\leq 0.001$. Each point is the average of three repeats for permeabilization or four repeats for cell toxicity where error bars show standard deviation. Error bars for $\mathbf{h}$ show $95 \% \mathrm{Cl}$.

interactions with the NTD/NAC) is accompanied by saturation of elongation. Elongation can be described as a two-step process, with monomer attachment to the fibril end, followed by conversion into the fibril structure. A saturation phenomenon with increasing levels of truncation could arise either from changes in binding $\left(K_{E}\right)$ or conversion $\left(k_{\mathrm{c}}\right)$. Despite the observed decrease in $K_{E}$ upon truncation, there is a slight decrease in the overall rate of elongation $k_{+}$. This implies that the conversion step $\left(k_{\mathrm{c}}\right)$ is slowed significantly by truncation.

Saturation of elongation of shorter constructs might be induced by a change in the structure of the formed fibril, leading to slower conversion. A CTD-truncated aSN lacking residues 109-140 produces more twisted fibrils with greater distance between the $\beta$ sheets and higher solvent exposure in comparison with $\alpha \mathrm{SN}-\mathrm{FL}$ fibers $^{24}$. Moreover, CTD-truncated fibrils do not elongate aSN-FL

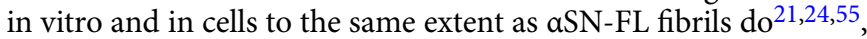
supporting a change in fibril structure. In contrast, monomers of truncated $\alpha \mathrm{SN}$ in a mixture with $\alpha \mathrm{SN}-\mathrm{FL}$ monomer accelerate fibrillation of aSN-FL in vitro and in cells ${ }^{21}$. We recapitulate this in our study, where $10 \%$ of CTD truncated mutant significantly accelerates aSN fibrillation. Even small amounts of truncated $\alpha \mathrm{SN}$ significantly increase production of seeds to make new fibrils and propagate disease. Thus, as implied elsewhere ${ }^{21}$, CTD truncated aSN monomers may promote spread of PD.

CTD truncation and His50 protonation both compromise oligomerization. Long-range interaction between the CTD and NAC/NTD domains of aSN might also be important for formation of aSN oligomers, since aSN121 and shorter mutants, which weaken or remove such interactions, fail to oligomerize. The inability to oligomerize at and below $\mathrm{pH} 6$ points to a critical protonation around this $\mathrm{pH}$ value. The only candidate is the single His residue in aSN (His50). Remarkably, aSN H50A was unable to oligomerize between $\mathrm{pH} 6$ and 9.5 (Fig. S8g), implying a critical role for a deprotonated imidazole ring in oligomerization. The basis for this remains unclear in the absence of a high-resolution (or residue-specific) oligomer structure. We note that residue 50 is part of a region of aSO which forms relatively stable secondary structure according to hydrogen exchange $\mathrm{MS}^{5}$, though the key structured region is centred around residues 70-88 according to solid state $\mathrm{NMR}^{56}$.

It is noteworthy that ONE allows all aSN constructs to oligomerize. ONE is a product of peroxidation of polyunsaturated fatty acids present in the cell membrane ${ }^{57}$, which has been reported to cross-link brain proteins ${ }^{58}$. In particular, ONE can induce aSN oligomer formation which could be physiologically relevant considering the role of oxidative stress in $\mathrm{PD}^{34,59}$. Therefore, we investigated ONE-induced oligomer formation in CTD truncated mutants that do not form oligomers on their own. ONE oligomers are more stable against dissociation in SDS or urea than normal oligomers ${ }^{34}$. ONE mostly modifies Lys residues and can thus crosslink different aSN molecules covalently ${ }^{34,60}$. ONE oligomers of shorter constructs are more elongated with relatively bigger shells than oligomers of aSN-FL (whether ONE modified or not). These oligomer properties illustrate how CTD truncation divides aSN constructs into two groups with different physiological properties. It is remarkable that ONE induces oligomer formation even in shorter constructs which are normally not able to form oligomer. Given that ONE-oligomers and unmodified oligomers have similar size and shape according to SAXS, we conclude that ONE helps otherwise unstable truncated oligomers form, instead of directing aSNs towards a different class of oligomers. In other words, ONE could be seen as filling in CTD's role in stabilizing the oligomer. The basis for this remains unclear. One speculative possibility is that just as the CTD's anionic tail may reduce the electrostatic repulsion caused by the more cationic N-terminal region, similarly ONE may overcome the N-terminal positive charges either by modifying the Lys side chains or by cross-linking them to other parts of the protein. If inter-molecular interactions between CTD and NTD in the oligomeric state indeed helps form and stabilize the oligomers, then conversely, shortening of the CTD will decrease 
the frequency and strength of these interactions, leading to extended tails and therefore oligomers with bigger shells. However, there is a limit to the ability of ONE to compensate for truncation since removal of sufficient residues (around aSN115) completely abolishes the ability to form oligomers without help from ONE.

Finally, the properties of aSOs are reflected in their ability to disrupt membranes and kill cells. Although the membrane penetration of monomeric aSN decreases gradually as CTD is truncated, oligomers of these mutants again fall into two groups: aSOs of shorter constructs with less membrane penetration ability and cell toxicity and aSOs of longer constructs with higher membrane disruption power and cell toxicity. Only NTD and NAC regions are known to interact directly with vesicles in monomeric aSN ${ }^{61,62}$, but truncation of CTD may have indirect effects by altering the core-shell distribution within the oligomer. A structural study on $\alpha \mathrm{SO}$ formation and membrane penetration showed a division of labor in which the NTD provides interfacial contacts while the $\beta$-sheet-rich core penetrates into the membrane ${ }^{56}$. Even though CTD truncation provides greater freedom for the NTD, a thicker shell covering the $\beta$-sheet core (a consequence of CTD truncation) may restrict the $\beta$-sheet core's membrane penetration and thus reduce cell toxicity.

In summary, C-terminal truncation of $\alpha \mathrm{SN}$ results in a very sudden increase in the rate of fibril replication upon truncation beyond residue 121 due to an increase in the secondary processes of fibrillation., most likely fragmentation This effect is apparent even when only $10 \%$ of the aggregating protein present is the truncated variant. By contrast, truncation beyond residue 121 essentially abolishes oligomerization. Taken together, our results show that though outside the actual aggregate core, residues in the region of residue 121 onwards act as an electrostatic doubleedged safety guard, supressing fibrillation while being prerequisites for oligomerization. Several CTD truncated $\alpha \mathrm{SN}$ s have been found in neural cells or Lewy bodies ${ }^{15,63}$. Our work identifies a potential "danger zone" of particularly deleterious CTD truncations, namely a CTD truncation range from aSN 121 to aSN 125 which shows increased fibrillation compared to aSNFL while retaining the ability to form cytotoxic aSN oligomers on their own. In this context it is striking that the most common in vivo truncated forms of aSN are aSN119 and aSN122 ${ }^{11}$. This highlights the deleterious consequences of biological processing of $a \mathrm{SN}$.

\footnotetext{
Methods

Protein purification and sample preparation. All proteins were expressed in $E$. coli BL21 (DE3) cells. aSN FL (human wild-type aSN) was expressed with pE11-D plasmid while all $\alpha \mathrm{SN}$ mutants were expressed with a pET15-B plasmid with a stop codon before the His-tag. All plasmids were transformed by electroporation and expressed using autoinduction media as described ${ }^{64}$. Constructs $\alpha \mathrm{SN} 121$ and longer (whose net charge is negative at $\mathrm{pH} 7.4$ ) were purified on an anion exchange column (HiTrap ${ }^{\mathrm{TM}} \mathrm{Q}$ HP) while constructs $\mathrm{aSN} 115$ and shorter (whose net charge is positive at $\mathrm{pH}$ 7.4) were purified on a cation exchange column (HiTrap ${ }^{\mathrm{TM}} \mathrm{SP}$ $\mathrm{HP}$ ). Purified protein was dialyzed in MQ water and lyophilized. Just prior to use, the lyophilized protein was dissolved in PBS $(13 \mathrm{mM}$ phosphate, $137 \mathrm{mM} \mathrm{NaCl}$, $3 \mathrm{mM} \mathrm{KCl}, \mathrm{pH} 7.4$ ) and filtered through a $0.2 \mu \mathrm{m}$ nitrocellulose membrane filter to remove any pre-existing aggregates. Protein concentration was measured on a Nanodrop 1000 spectrophotometer (Thermo Scientific) using theoretical extinction coefficients at $280 \mathrm{~nm}$ based on the content of Tyr residues (between 1 and 4 depending on construct).
}

ThT fibrillation assays. Fibrillation kinetics was monitored by Thioflavin- $\mathrm{T}$ (ThT) fluorescent assay in black polystyrene 96-well plate using a TECAN Infinite M200 microplate reader pre-adjusted to $37^{\circ} \mathrm{C}$. 3 -mm glass beads were used for experiments with shaking ( $300 \mathrm{rpm}, 10 \mathrm{~min}$ shaking in $12 \mathrm{~min}$ measurement intervals). Shaking for low-seed fibrillation assay was done only $20 \mathrm{~s}$ in every $10 \mathrm{~min}$ measurement intervals. Freshly sonicated fibrils (probe sonicated for $15 \mathrm{~s}$ ) were used for seeding experiment (performed in quiescent mode). $40 \mu \mathrm{M}$ ThT was used for all experiments.

\section{Analysis of fibrillation kinetics}

Description of model. Normalized ThT fibrillation data were fitted to a molecular mechanism of filamentous aggregation that includes primary nucleation, frag mentation, and saturating elongation. The differential equations describing the time evolution of aggregate number concentration, $\mathrm{P}(\mathrm{t})$, and aggregate mass concentration, $\mathrm{M}(\mathrm{t})$, are

$$
\begin{aligned}
& \frac{d P}{d t}=k_{n} m(t)^{n_{c}}+k_{-} M(t) \\
& \frac{d M}{d t}=2 k_{+} \frac{m(t)}{\left(1+\frac{m(t)}{K_{E}}\right)} P(t)
\end{aligned}
$$

where $k_{n}, k_{-}$and $k_{+}$are the rate constants of primary nucleation, fragmentation and elongation, $K_{E}$ is the saturation concentration of elongation, $m(t)$ is the monomer concentration at time $t$ and $n_{c}$ is the reaction order of primary nucleation. Approximate analytical solutions to these equations, the integrated rate laws ${ }^{39}$, are used to fit the data. Note that if secondary nucleation instead of fragmentation was the dominant process by which new fibrils are formed, $k_{\text {- }}$ in the above equation would be replaced by $k_{2} m(t)^{n 2}$, where $k_{2}$ and $n_{2}$ are the rate constant and reaction order of secondary nucleation. When $n_{2}=0$, the two expressions are mathematically equivalent, making it impossible to distinguish fragmentation from saturated secondary nucleation based on fits of $M(t)$ and $P(t)$ alone ${ }^{40}$. A detailed investigation to distinguish fragmentation from secondary nucleation in $\alpha \mathrm{SN}$ aggregation at low $\mathrm{pH}$ can be found in Gaspar et al. ${ }^{41}$

Effective elongation rates. These were obtained using ThT fibrillation assays with $50 \mu \mathrm{M}$ of $\alpha \mathrm{SN}$ monomer and $2.5-12.5 \mu \mathrm{M}$ seeds (monomer units), corresponding to $5-25 \%$ of monomer. To calculated the effective rate of elongation ${ }^{65}$, initial rates of fibrillation were plotted against the seed concentration and the slope of the fitted line was used to determine the effective elongation rate. The initial rate of increase in the presence of significant amounts of seeds is approximated by:

$$
\frac{d M}{d t}=2 k_{+} \frac{m_{0}}{\left(1+\frac{m_{0}}{K_{E}}\right)} P_{0}
$$

where $m_{0}$ is the initial monomer concentration and $P_{0}$ is the number concentration of seed fibrils. Both $m_{0}$ and $K_{E}$ are known, the latter from the fits of the unseeded data as described above, so we can determine the product $P_{0} k_{+}$. However, the initial seed number concentration, $P_{0}$, (not to be confused with the seed mass concentration $M$ ) is difficult to determine accurately. We are here interested in the relative variations of the rates as the degree of truncation changes, so rather than estimate $P_{0}$ to obtain absolute values for $k_{+}$, we instead compare the relative values of $P_{0} k_{+}$, setting the value for the full-length protein to 1 . Under the assumption that the length of the sonicated seeds does not vary with truncation, relative changes in $P_{0} k_{+}$are equivalent to relative changes in the elongation rate constant $k_{+}$

Oligomer preparation. To increase yields of $\alpha \mathrm{SN}$ oligomers, each $\alpha \mathrm{SN}$ construct (initially lyophilized after purification as described above) was subjected to two cycles of dissolution to $\sim 10 \mathrm{mg} / \mathrm{ml}$ in milliQ followed by freeze-drying. Subsequently, the construct was dissolved to $8 \mathrm{mg} / \mathrm{mL}$ in PBS and incubated at $37^{\circ} \mathrm{C}$ for $4 \mathrm{~h}$ in 1.5 microtubes while shaking at $900 \mathrm{rpm}$. Large aggregates were spun down at $12,000 \times g$ for $5 \mathrm{~min}$. The supernatant was applied on a SEC column (Superose 6 Prep Grade column; GE Healthcare Life Sciences, Sweden) by AKTA system (GE Healthcare) and oligomer fractions were collected for further analysis. $100-\mathrm{kDa}$ centrifuge filters $\left(\right.$ Amicon $^{\circledR}$ ) were used to concentrate the collected oligomers. To prepare oligomers in different $\mathrm{pH}$ and salt concentrations, monomers were first dissolved in milliQ water and then diluted 1:1 to the desired working concentration with $2 \times$ concentrated buffers.

DMPG vesicle preparation. DMPG vesicles were prepared as described ${ }^{66}$. Briefly, $2 \mathrm{mg}$ of 1,2-dimyristoyl-sn-3-phosphatidylglycerol (DMPG) was suspended in $1 \mathrm{~mL}$ PBS and freeze-thawed in liquid nitrogen and a $55^{\circ} \mathrm{C}$ water bath respectively, 10 times. The solution was extruded 21 times through a $200 \mathrm{~nm}$ filter (Whatman, Nuclepore TE membrane). To prepare calcein-filled vesicles, $10 \mathrm{mg}$ DMPG was suspended in $1 \mathrm{~mL}$ PBS including $70 \mathrm{mM}$ calcein with the same procedure as normal vesicle preparation. Then, the excess calcein was removed by PD-10 desalting column (GE Healthcare) and the fractions containing calcein were identified by monitoring fluorescence (VarioScan Flash fluorimeter (Thermofisher Scientific); excitation at 495 and emission at $515 \mathrm{~nm}$ with a $5 \mathrm{~nm}$ bandwidth) after adding Triton X-100. Vesicles were prepared and used within 1 day.

CD spectroscopy. Far-UV CD spectra were recorded on a Jasco J-810 spectrophotometer (Jasco Spectroscopic Co. Ltd., Japan) from 250 to $195 \mathrm{~nm}$ using $0.2 \mathrm{mg} / \mathrm{ml}$ of aSN monomers or oligomers in a $1 \mathrm{~mm}$ cuvette.

Small-angle X-ray scattering (SAXS). SAXS was carried out on an optimized NanoSTARSAXS instrument ${ }^{67}$ from Bruker AXS at Aarhus University equipped 
with scatterless slits ${ }^{68}$ and a liquid Ga metal jet X-ray source from Excillum ${ }^{69}$, which measures at a wavelength of $\lambda=1.34 \AA$. The SUPERSAXS program package (Oliveira, C.L.P. and Pedersen J.S., un-published) was used for background subtraction and to convert data to absolute scale. Water was used as a calibration standard and the data is plotted as a function of the scattering vector $q=4 \sin (\theta) / \lambda$. SEC-purified oligomers were measured at a concentration ranging from 0.19 to $1.35 \mathrm{mg} / \mathrm{mL}$ depending on the yield and were measured for $30 \mathrm{~min}$ at $20^{\circ} \mathrm{C}$. Model independent information of the oligomers was obtained by the Indirect Fourier Transformation (IFT) procedure ${ }^{70}$ that gives the pair distance distribution function $p(r)$ that gives real-space distance information. To obtain further insight in the oligomer structure, we modeled the data with a previously developed aSN oligomer model $^{66}$ where the oligomer is assumed to have a prolate ellipsoid shape with a compact core and a shell of flexible polymer-like protein. By modeling the data on absolute scale, the average number of monomers in each complex $\left(N_{\text {chain }}\right)$ can be obtained. Furthermore, the long $\left(r_{\text {long }}\right)$ and short $\left(r_{\text {short }}\right)$ axis of the core, the thickness of the shell $\left(D_{\text {shell }}\right)$ and the number of amino acids in in the shell can be modeled.

Transmission electron microscopy (TEM). $5 \mu \mathrm{L}$ of $0.2 \mathrm{mg} / \mathrm{mL}$ oligomer or $0.4 \mathrm{mg} / \mathrm{mL}$ fibril solution was applied to glow-discharged 400 -mesh carbon-coated copper grids for $60 \mathrm{~s}$ before being removed with filter paper and immediately stained with $3 \mu \mathrm{L}$ of a $2 \%(\mathrm{w} / \mathrm{v})$ aqueous uranyl formate solution for $15 \mathrm{~s}$. The grid was stained again for $15 \mathrm{~s}$ before excess stain was removed by blotting with filter paper. TEM was performed on a Tecnai ${ }^{\text {tw }}$ G2 Spirit transmission electron microscope operated at $120 \mathrm{kV}$. Digital acquisitions were performed with a bottommounted TVIPS camera.

Calcein release assay. Calcein release was performed in triplicates and in the range of 0.001 to $100 \mu \mathrm{M}$ of monomers and 0.00005 to $10 \mu \mathrm{M}$ of oligomers as described ${ }^{66}$. Briefly, $2 \mu \mathrm{L}$ of vesicle was added to $138 \mu \mathrm{L}$ PBS in a 96-well plate and then $10 \mu \mathrm{L}$ of protein solution was added to each well. The fluorescence of calcein (excitation at 495 and emission at $515 \mathrm{~nm}$ ) was monitored by VarioScan Flash fluorimeter (Thermofisher Scientific) using a $5 \mathrm{~nm}$ bandwidth. After $1 \mathrm{~h}, 2 \mu \mathrm{L}$ of Triton X-100 was added to each well to lyse all vesicles and release all calcein. Calcein release (CR) was calculated according to:

$$
C R \%=100 \times\left(F_{p}-F_{c}\right) /\left(F_{f}-F_{c}\right)
$$

where $F_{\mathrm{p}}$ and $F_{\mathrm{c}}$ are the fluorescence intensities after $1 \mathrm{~h}$ incubation with $\alpha \mathrm{SN}$ and PBS only, respectively, (either monomer or oligomer) and $F_{\mathrm{f}}$ is the fluorescence intensity after addition of Triton X-100. The data were plotted in KaleidaGraph software (V 4.5) and fitted with a sigmoidal model. $\mathrm{CR}^{50 \%}$ is the concentration of protein required to disrupt $50 \%$ of vesicles.

Cell toxicity assay. MTT (3-[4,5-dimethylthiazol-2-yl] -2,5-diphenyltetrazolium bromide) assay was used to measure the cell viability after $24 \mathrm{~h}$ treatment with different variants of C-terminally truncated aSN oligomers. SH-SY5Y cells were seeded in 96-well plates at a density of $5 \times 10^{4} \mathrm{cell} / \mathrm{ml}$ in DMEM media supplemented with $10 \%$ FBS, 100 units $/ \mathrm{ml}$ penicillin and $100 \mu \mathrm{g} / \mathrm{ml}$ streptomycin. The cells were incubated in a humidified atmosphere incubator with $5 \% \mathrm{CO}_{2}$ and $95 \%$ humidity at $37^{\circ} \mathrm{C}$ for $24 \mathrm{~h}$. The culture medium was then replaced with fresh medium containing $10 \mu \mathrm{M} \alpha$-SN oligomers and incubated for another $24 \mathrm{~h}$. Then the cell medium was replaced with fresh medium containing $20 \%$ MTT $(5 \mathrm{mg} / \mathrm{ml})$ and the cells were incubated for an additional $4 \mathrm{~h}$ at $37^{\circ} \mathrm{C}$. Subsequently $100 \mu \mathrm{l}$ DMSO was added to dissolve formazan crystals by incubating for $30 \mathrm{~min}$ on a shaker at room temperature. Absorbance was measured on a plate reader at $570 \mathrm{~nm}$.

Statistics and Reproducibility. Every experiment generally tests different concentrations with three repeats. In Fig. 1, every data points correspond to a repeat. In Fig. $2 \mathrm{a}-\mathrm{c}$, every data point corresponds to a separate experiment with a series of concentrations, while in Fig. $2 \mathrm{e}-\mathrm{g}$, curves are the average of two repeats. SAXS experiment was done once for each oligomer. Calcein release experiment has been done with three repeats and cell toxicity with 4 repeats. All the error bars show standard deviation except for Fig. $4 \mathrm{~h}$ which is $95 \%$ confidence interval (CI). Oneway ANOVA was performed for comparisons in Fig. 4h using GraphPad prism version 8 (GraphPad Software, San Diego, California USA).

Reporting summary. Further information on research design is available in the Nature Research Reporting Summary linked to this article.

\section{Data availability}

Source data for graphs and charts in the main figures is provided as Supplementary Data 1 and any remaining information can be obtained from the corresponding author upon reasonable request. Supplementary Data 1.xlsx file include 8 data sheets named after main figures and panels.

\section{Code availability}

No custom code was used in this work.
Received: 15 June 2021; Accepted: 18 January 2022;

Published online: 10 February 2022

\section{References}

1. Stefanis, L. alpha-Synuclein in Parkinson's disease. Cold Spring Harb. Perspect. Med. 2, a009399 (2012).

2. Volles, M. J. \& Lansbury, P. T. Zeroing in on the pathogenic form of alphasynuclein and its mechanism of neurotoxicity in Parkinson's Disease. Biochemistry 42, 7871-7878 (2003).

3. Reynolds, N. P. et al. Mechanism of membrane interaction and disruption by alpha-synuclein. J. Am. Chem. Soc. 133, 19366-19375 (2011).

4. Huang, M. et al. A-Synuclein: A multifunctional player in exocytosis, endocytosis, and vesicle recycling. Front. Neurosci. 13, 1-8 (2019).

5. Paslawski, W., Mysling, S., Thomsen, K., Jørgensen, T. J. D. \& Otzen, D. E. Coexistence of two different $\alpha$-synuclein oligomers with different core structures determined by hydrogen/deuterium exchange mass spectrometry. Angew. Chem. Int Ed. Engl. 53, 7560-7563 (2014).

6. Tuttle, M. D. et al. Solid-state NMR structure of a pathogenic fibril of full-length human alpha-synuclein. Nat. Struct. Mol. Biol. 23, 409-415 (2016).

7. Li, B. et al. Cryo-EM of full-length alpha-synuclein reveals fibril polymorphs with a common structural kernel. Nat. Commun. 9, 3609 (2018).

8. Lorenzen, $N$. et al. How epigallogatechin gallate can inhibit a-synuclein oligomer toxicity in vitro. J. Biol. Chem. 289, 21299-21310 (2014).

9. Lautenschlager, J. et al. C-terminal calcium binding of alpha-synuclein modulates synaptic vesicle interaction. Nat. Commun. 9, 712 (2018).

10. Zhang, Z. et al. Calcium accelerates SNARE-mediated lipid mixing through modulating a-synuclein membrane interaction. Biochimica et. Biophysica Acta Biomembr. 1860, 1848-1853 (2018).

11. Sorrentino, Z. A. \& Giasson, B. I. The emerging role of a-synuclein truncation in aggregation and disease. J. Biol. Chem. 295, 10224-10244 (2020).

12. Liu, C. W. et al. A precipitating role for truncated $\alpha$-synuclein and the proteasome in $a$-synuclein aggregation: Implications for pathogenesis of parkinson disease. J. Biol. Chem. 280, 22670-22678 (2005).

13. Mishizen-Eberz, A. J. et al. Distinct cleavage patterns of normal and pathologic forms of $\alpha$-synuclein by calpain I in vitro. J. Neurochem. 86, 836-847 (2003).

14. Nishijima David, L., Wisner, D. H., Holmes, J. F. D. \& Simel, K. Oxidants Induce Alternative Splicing Of A-Synuclein: Implications For Parkinson's Disease. Physiol. Behav. 176, 139-148 (2016).

15. Li, W. et al. Aggregation promoting C-terminal truncation of $\alpha$-synuclein is a normal cellular process and is enhanced by the familial Parkinson's disease-linked mutations. Proc. Natl Acad. Sci. USA 102, 2162-2167 (2005).

16. Ulusoy, A., Febbraro, F., Jensen, P. H., Kirik, D. \& Romero-Ramos, M. Coexpression of C-terminal truncated alpha-synuclein enhances full-length alpha-synuclein-induced pathology. Eur. J. Neurosci. 32, 409-422 (2010).

17. Games, D. et al. Reducing C-terminal-truncated alpha-synuclein by immunotherapy attenuates neurodegeneration and propagation in Parkinson's disease-like models. J. Neurosci. 34, 9441-9454 (2014).

18. Levitan, K. et al. Conserved C-terminal charge exerts a profound influence on the aggregation rate of a-synuclein. J. Mol. Biol. 411, 329-333 (2011).

19. Van Der Wateren, I. M., Knowles, T. P. J., Buell, A. K., Dobson, C. M. \& Galvagnion, C. C-terminal truncation of a-synuclein promotes amyloid fibril amplification at physiological pH. Chem. Sci. 9, 5506-5516 (2018).

20. Hoyer, W., Cherny, D., Subramaniam, V. \& Jovin, T. M. Impact of the acidic C-terminal region comprising amino acids 109-140 on $\alpha$-synuclein aggregation in vitro. Biochemistry 43, 16233-16242 (2004).

21. Sorrentino, Z. A. et al. Physiological C-terminal truncation of a-synuclein potentiates the prion-like formation of pathological inclusions. J. Biol. Chem. 293, 18914-18932 (2018).

22. Dedmon, M. M., Lindorff-Larsen, K., Christodoulou, J., Vendruscolo, M. \& Dobson, C. M. Mapping long-range interactions in $\alpha$-synuclein using spinlabel NMR and ensemble molecular dynamics simulations. J. Am. Chem. Soc. 127, 476-477 (2005).

23. Bernadó, P., Bertoncini, C. W., Griesinger, C., Zweckstetter, M. \& Blackledge, M. Defining long-range order and local disorder in native a-synuclein using residual dipolar couplings. J. Am. Chem. Soc. 127, 17968-17969 (2005).

24. Iyer, A. et al. C-Terminal truncated $\alpha$-synuclein fibrils contain strongly twisted $\beta$-sheets. J. Am. Chem. Soc. 139, 15392-15400 (2017).

25. Ni, X., McGlinchey, R. P., Jiang, J. \& Lee, J. C. Structural insights into alphasynuclein fibril polymorphism: effects of Parkinson's disease-related Cterminal truncations. J. Mol. Biol. 431, 3913-3919 (2019).

26. Winner, B. et al. In vivo demonstration that -synuclein oligomers are toxic. Proc. Natl Acad. Sci. USA 108, 4194-4199 (2011). 
27. Yamamoto, K., Izumi, Y., Arifuku, M., Kume, T. \& Sawada, H. a-Synuclein oligomers mediate the aberrant form of spike-induced calcium release from IP3 receptor. Sci. Rep. 9, 15977-15977 (2019).

28. Andreasen, M., Lorenzen, N. \& Otzen, D. E. Interactions between misfolded protein oligomers and membranes: a central topic in neurodegenerative diseases? Biochim. Biophys. Acta 1848, 1897-1907 (2015).

29. van Diggelen, F., Tepper, A. W. J. W., Apetri, M. M. \& Otzen, D. E. asynuclein oligomers: a study in diversity. Isr. J. Chem. 57, 699-723 (2017).

30. Zijlstra, N., Blum, C., Segers-Nolten, I. M. J., Claessens, M. M. A. E. \& Subramaniam, V. Molecular composition of sub-stoichiometrically labeled $a-$ synuclein oligomers determined by single-molecule photobleaching. Angew. Chem. Int. Ed. 51, 8821-8824 (2012).

31. Paslawski, W., Mysling, S., Thomsen, K., Jørgensen, T. J. D. \& Otzen, D. E. Co-existence of two different $a$-synuclein oligomers with different core structures determined by hydrogen/deuterium exchange mass spectrometry. Angew. Chem. Int. Ed. 53, 7560-7563 (2014).

32. Lorenzen, $\mathrm{N}$. et al. The role of stable $\alpha$-synuclein oligomers in the molecular events underlying amyloid formation. J. Am. Chem. Soc. 136, 3859-3868 (2014).

33. Paslawski, W. et al. High stability and cooperative unfolding of cytotoxic asynuclein oligomers. Biochemistry 53, 6252-6263 (2014).

34. Almandoz-Gil, L. et al. Low molar excess of 4-oxo-2-nonenal and 4-hydroxy2-nonenal promote oligomerization of alpha-synuclein through different pathways. Free Radic. Biol. Med. 110, 421-431 (2017).

35. Paik, S. R., Shin, H. J., Lee, J. H., Chang, C. S. \& Kim, J. Copper(II)-induced self-oligomerization of alpha-synuclein. Biochem J. 340, 821-828 (1999).

36. Lowe, R., Pountney, D. L., Jensen, P. H., Gai, W. P. \& Voelcker, N. H. Calcium(II) selectively induces alpha-synuclein annular oligomers via interaction with the C-terminal domain. Protein Sci. 13, 3245-3252 (2004).

37. Rekas, A. et al. The structure of dopamine induced alpha-synuclein oligomers. Eur. Biophys. J. 39, 1407-1419 (2010).

38. Mazzulli, J. R., Armakola, M., Dumoulin, M., Parastatidis, I. \& Ischiropoulos, $\mathrm{H}$. Cellular oligomerization of alpha-synuclein is determined by the interaction of oxidized catechols with a C-terminal sequence. J. Biol. Chem 282, 31621-31630 (2007)

39. Meisl, G. et al. Molecular mechanisms of protein aggregation from global fitting of kinetic models. Nat. Protoc. 11, 252-272 (2016).

40. Meisl, G. et al. Scaling behaviour and rate-determining steps in filamentous self-assembly. Chem. Sci. 8, 7087-7097 (2017).

41. Gaspar, R. et al. Secondary nucleation of monomers on fibril surface dominates alpha-synuclein aggregation and provides autocatalytic amyloid amplification. Q Rev. Biophys. 50, e6 (2017).

42. Hill, E. K., Krebs, B., Goodall, D. G., Howlett, G. J. \& Dunstan, D. E. Shear flow induces amyloid fibril formation. Biomacromolecules 7, 10-13 (2006).

43. Morinaga, A. et al. Critical role of interfaces and agitation on the nucleation of $\mathrm{A} \beta$ amyloid fibrils at low concentrations of $\mathrm{A} \beta$ monomers. Biochim. Biophys. Acta https://doi.org/10.1016/j.bbapap.2010.01.012 (2010).

44. Giehm, L. \& Otzen, D. E. Strategies to increase the reproducibility of protein fibrillization in plate reader assays. Anal. Biochem. 400, 270-281 (2010).

45. Stephens, A. D. et al. Different structural conformers of monomeric $\alpha-$ synuclein identified after lyophilizing and freezing. Anal. Chem. 90, 6975-6983 (2018).

46. Danzer, K. M. et al. Different species of alpha-synuclein oligomers induce calcium influx and seeding. J. Neurosci. 27, 9220-9232 (2007).

47. Feng, L. R., Federoff, H. J., Vicini, S. \& Maguire-Zeiss, K. A. Alpha-synuclein mediates alterations in membrane conductance: a potential role for alphasynuclein oligomers in cell vulnerability. Eur. J. Neurosci. 32, 10-17 (2010).

48. Flagmeier, P. et al. Ultrasensitive Measurement of $\mathrm{Ca}(2+)$ Influx into Lipid Vesicles Induced by Protein Aggregates. Angew. Chem. Int Ed. Engl. 56, 7750-7754 (2017).

49. Izawa, Y. et al. Role of C-terminal negative charges and tyrosine residues in fibril formation of alpha-synuclein. Brain Behav. 2, 595-605 (2012)

50. Stephens, A. D. et al. Extent of N-terminus exposure of monomeric alphasynuclein determines its aggregation propensity. Nat. Commun. 11, 2820 (2020).

51. Okazaki, H. et al. Remaining structures at the $\mathrm{N}$ - and C-terminal regions of alpha-synuclein accurately elucidated by amide-proton exchange NMR with fitting. FEBS Lett. 587, 3709-3714 (2013).

52. Dass, R., Corlianò, E. \& Mulder, F. A. A. The contribution of electrostatics to hydrogen exchange in the unfolded protein state. Biophys. J. https://doi.org/ 10.1016/j.bpj.2021.08.003 (2021).

53. Jha, N. N. et al. Complexation of NAC-derived peptide ligands with the C-terminus of alpha-synuclein accelerates its aggregation. Biochemistry 57, 791-804 (2018).

54. Hong, D. P., Xiong, W., Chang, J. Y. \& Jiang, C. The role of the C-terminus of human alpha-synuclein: intra-disulfide bonds between the C-terminus and other regions stabilize non-fibrillar monomeric isomers. FEBS Lett. $\mathbf{5 8 5}$, 561-566 (2011).
55. Sorrentino, Z. A., Xia, Y., Gorion, K. M., Hass, E. \& Giasson, B. I. Carboxyterminal truncations of mouse alpha-synuclein alter aggregation and prionlike seeding. FEBS Lett. 594, 1271-1283 (2020).

56. Fusco, G. et al. Structural basis of membrane disruption and cellular toxicity by alpha-synuclein oligomers. Science 358, 1440-1443 (2017).

57. Lee, S. H. \& Blair, I. A. Characterization of 4-Oxo-2-nonenal as a novel product of lipid peroxidation. Chem. Res. Toxicol. 13, 698-702 (2000).

58. Stewart, B. J., Doorn, J. A. \& Petersen, D. R. Residue-specific adduction of tubulin by 4-hydroxynonenal and 4-oxononenal causes cross-linking and inhibits polymerization. Chem. Res. Toxicol. 20, 1111-1119 (2007).

59. Näsström, T. et al. The lipid peroxidation products 4-oxo-2-nonenal and 4 hydroxy-2-nonenal promote the formation of $\alpha$-synuclein oligomers with distinct biochemical, morphological, and functional properties. Free Radic. Biol. Med. 50, 428-437 (2011).

60. Zhu, X. \& Sayre, L. M. Long-lived 4-oxo-2-enal-derived apparent lysine michael adducts are actually the isomeric 4-ketoamides. Chem. Res Toxicol. 20, 165-170 (2007)

61. Fusco, G. et al. Direct observation of the three regions in a-synuclein that determine its membrane-bound behaviour. Nat. Commun. 5, 3827 (2014).

62. Antonschmidt, L. et al. Insights into the molecular mechanism of amyloid filament formation: Segmental folding of alpha-synuclein on lipid membranes. Sci. Adv. 7, https://doi.org/10.1126/sciadv.abg2174 (2021).

63. Pieri, L. et al. Cellular response of human neuroblastoma cells to $\alpha$-synuclein fibrils, the main constituent of Lewy bodies. Biochim. Biophys. Acta 1860, 8-19 (2016).

64. Mohammad-Beigi, H. et al. Oleuropein derivatives from olive fruit extracts reduce alpha-synuclein fibrillation and oligomer toxicity. J. Biol. Chem. 294, 4215-4232 (2019).

65. Zaman, M. \& Andreasen, M. Cross-talk between individual phenol soluble modulins in $S$. aureus biofilm enables rapid and efficient amyloid formation. eLife 9, https://doi.org/10.7554/eLife.59776 (2020).

66. Sahin, C. et al. a-Synucleins from animal species show low fibrillation propensities and weak oligomer membrane disruption. Biochemistry 57, 5145-5158 (2018)

67. Pedersen, J. A flux- and background-optimized version of the NanoSTAR small-angle X-ray scattering camera for solution scattering. J. Appl. Crystallogr. 37, 369-380 (2004)

68. Li, Y., Beck, R., Huang, T., Choi, M. C. \& Divinagracia, M. Scatterless hybrid metal-single-crystal slit for small-angle X-ray scattering and high-resolution X-ray diffraction. J. Appl. Crystallogr. 41, 1134-1139 (2008).

69. Schwamberger, A. et al. Combining SAXS and DLS for simultaneous measurements and time-resolved monitoring of nanoparticle synthesis. $\mathrm{Nucl}$. Instrum. Methods Phys. Res. Sect. B: Beam Interact. Mater. At. 343, 116-122 (2015).

70. Glatter, O. A new method for the evaluation of small-angle scattering data. J. Appl. Crystallogr. 10, 415-421 (1977).

\section{Acknowledgements}

D.E.O. (grant R276-2018-671) and A.K.S. (grant R287-2018-1836) are grateful to the Lundbeck Foundation for generous and sustained support. We also thank the Novo Nordisk Foundation for supporting D.E.O. and P.A. (grant NNF17OC0028806). We thank Dr. Frans Mulder for inspiring discussions about aSN electrostatics.

\section{Author contributions}

A.F. and D.E.O. conceived the project. A.F. carried out all protein purification and aggregation studies, performed all kinetic analysis and wrote the first draft. D.E.O. supervised all aspects of the project, obtained funding, carried out project administration and edited the manuscript. J.N.P. and J.S.P. carried out SAXS experiments and analysis G.M. and T.K. helped analyse kinetic data. A.K.S. carried out data analysis. P.A. measured cell viability. L.G. provided truncated aSN constructs, supervised by M.A.N and A.F.S.

\section{Competing interests}

The authors declare no competing interests.

\section{Additional information}

Supplementary information The online version contains supplementary material available at https://doi.org/10.1038/s42003-022-03059-8.

Correspondence and requests for materials should be addressed to Daniel Erik Otzen

Reprints and permission information is available at http://www.nature.com/reprints

Publisher's note Springer Nature remains neutral with regard to jurisdictional claims in published maps and institutional affiliations. 
(c) (i) Open Access This article is licensed under a Creative Commons Attribution 4.0 International License, which permits use, sharing, adaptation, distribution and reproduction in any medium or format, as long as you give appropriate credit to the original author(s) and the source, provide a link to the Creative Commons license, and indicate if changes were made. The images or other third party material in this article are included in the article's Creative Commons license, unless indicated otherwise in a credit line to the material. If material is not included in the article's Creative Commons license and your intended use is not permitted by statutory regulation or exceeds the permitted use, you will need to obtain permission directly from the copyright holder. To view a copy of this license, visit http://creativecommons.org/ licenses/by/4.0/.

(c) The Author(s) 2022 